\title{
Tunneling through a multigrain system: deducing the sample topology from the nonlinear conductance
}

\author{
Andrey V. Danilov ${ }^{a}$, Dmitrii S. Golubev ${ }^{b}$, and Sergey E. Kubatkin ${ }^{a}$ \\ MINA, Chalmers University of Technology, S-41296, Göteborg, Sweden \\ ${ }^{a}$ also at Kapitza Institute, 113117 Moscow, Russia \\ ${ }^{b}$ also at Lebedev Physics Institute, 117924, Moscow, Russia
}

We study a current transport through a system of a few grains connected with tunneling links. The exact solution is given for an arbitrarily connected double-grain system with a shared gate in the framework of the orthodox model. The obtained result is generalized for multigrain systems with strongly different tunneling resistances. We analyse the large-scale nonlinear conductance and demonstrate how the sample topology can be unambiguously deduced from the spectroscopy pattern (differential conductance versus gate-bias plot). We present experimental data for a multigrain sample and reconstruct the sample topology. A simple selection rule is formulated to distinguish samples with spectral patterns free from spurious disturbance caused by recharging of some grains nearby. As an example, we demonstrate experimental data with additional peaks in the spectroscopy pattern, which can not be attributed to coupling to additional grains. The described approach can be used to judge the sample topology when it is not guaranteed by fabrication and direct imaging is not possible.

PACS numbers: 73.23.Hk, 73.50.-h, 85.30.Vw 


\section{INTRODUCTION}

The electrical properties of nanostructures with ever-smaller size have been studied intensively during the past decade in a search for new quantum effects and novel types of electronic devices. The scientific interest has moved towards objects with sizes of a few nanometers. Such objects cannot be prepared by conventional electron beam lithography with a resolution being limited to $\sim 20 \mathrm{~nm}$. The laboratory methods, developed thus far, make use of prefabricated nanoscale objects: semiconductor [1] or metallic [2] nanocrystals made by colloidal chemistry, organometallic carborane clusters [3], and the fullerene molecules [4]. The granular films formed due to surface atom diffusion at room temperature [5 9] or in a process of an avalanche reconstruction of a quench-condensed precursor layer [10] were also used as a natural source of nanoparticles. These prefabricated nanoparticles have been spread on a surface and studied with STM [3]; or placed into a gap between two macroscopic electrodes defined by electron beam litography [1] or made by a break-junction technique [4].

The final sample geometry, i.e. the number of nanoparticles in a gap and their arrangement is not under experimental control. With some luck, one can get a sample where a single nanograin bridges two macroscopic electrodes. Typical yields of $25-30 \%$ have been reported [6.8.

Several methods to get a better defined sample geometry were suggested. A hole in a silicon-nitride membrane was used to restrict a contact area [5] [7. A self-assembling of gold clusters on electrodes covered with monolayers of organic molecules was used by Ref. [2]. An alternative way is to modify a sample by in situ material deposition, as described in 9.10 .

Nevertheless, not a single method ensures, just by the fabrication procedure itself, that there is no more than one nanoparticle in a gap. Moreover, even if the current mainly goes through a single grain, there may be some additional grains nearby which can potentially affect transport properties due to capacitance coupling. Sometimes, the sample geometry can be imaged directly by STM or SEM, like in [1,8], but sometimes not, as the samples are metastable and can only exist at cryogenic temperatures [10], or nanoobjects are simply too small, like the fullerene molecules [.

The only universal way to judge the sample topology is to analyze current transport data. Several criteria have been suggested in the literature, all based on the Coulomb blockade phenomena [11]. A nano-scale object, coupled to macroscopic source and drain electrodes, is a Single Electron Transistor - SET (although not every method allows to implement a third electrode - gate). The exact periodicity of the conductance variation versus bias and gate voltages in a single grain SET is violated if more than one grain is involved in the current transport [12]. So one can formulate an intuitive "selection rule" for single grain samples as "the ones showing Coulomb-staircase structure" [6,2,7] or, more precisely, "the ones with a single set of equally spaced Coulomb-staircase peaks in the differential conductance" [5].

Within the scope of the orthodox model for SET [11], the Coulomb staircases are the only peculiarities in the differential conductance. The real nanoscale samples, however, never follow this model exactly. First, if a typical level spacing $\delta$ between the quantised energy levels in a nanoparticle is comparable with the charging energy $E_{\mathrm{C}}$, then the Coulomb diamonds have different sizes and the Coulomb staircases are no more periodic [13]. Second, if the temperature is below the inter-level spacing $\delta$, then every discrete energy level in a grain gives an additional peak in the differential conductance $\mathrm{d} I / \mathrm{d} V$ [5]. In fact, it was the discrete excitation spectrum in a nanoparticle, which was the primary subject of the research in references [4 8 ].

Therefore, one should distinguish between the peculiarities coming from the discrete energy levels and a potential disturbance due to additional grains nearby [14. If all energy levels in the grain energy spectrum are equally shifted by the gate potential then any discrete energy state is revealed in a $\mathrm{d} I / \mathrm{d} V\left(V, V_{g}\right)$ plot $\left(V\right.$ is the gate and $V_{g}$ is the bias voltage) as a straight line parallel to the diamond edge [b]. This allows us to reformulate the selection rule for single grain samples as "the ones with all spectral lines in a $\mathrm{d} I / \mathrm{d} V\left(V, V_{g}\right)$ plot parallel to the diamond edges".

However, the gate voltage shifts all discrete levels the same way if only the external electrostatic field is effectively screened within a thin surface layer $\Delta \ll R$, where $R$ is characteristic sample radius. This is valid in the extreme case of a macroscopic metallic sample, but definitely does not take place for the opposite extreme case - a fullerene molecule. Moreover, additional spectral features can be expected due to excitation of mechanical degrees of freedom [4, 15], including the shuttle effects predicted in [16], and they could have a distinct gate dependency. To summarize, no single qualitative criterion, suggested thus far, provides a conclusive argument to prove a single grain sample topology.

In this paper we report the exact solution (within an orthodox model) for an arbitrary double grain system, and some mathematical results concerning the multigrain systems. Based on our analysis, we can formulate an excluding criterion, i.e. we can specify what kind of spectral pattern can never be the result of spurious disturbance from one or more additional grains nearby. We also present, as an example, experimental data of this type. 


\section{ENERGY DECREMENTS ON TUNNELING}

In our analysis we follow the solution of an orthodox model for a single-grain SET, as given in [11, with a proper generalisation for a double-grain system. Whenever possible, we also use the notations introduced in [11.

The electrostatic charging energy decrement $\Delta K_{\alpha, \beta}$ during the tunneling jump of an electron between the grain $\alpha$ and the lead electrode $\beta$ can be found directly and equals (see also Fig. 1 for notations):

$$
\begin{aligned}
\Delta \vec{K}_{\alpha \beta}=\frac{1}{C_{\Delta}^{2}}[ & e^{2}\left(C_{\Sigma \bar{\alpha}}\left((-1)^{\beta} n_{\alpha}-\frac{1}{2}\right)+C(-1)^{\beta} n_{\bar{\alpha}}\right)+ \\
& \left.+e V\left(C_{\Sigma \bar{\alpha}} C_{\alpha \bar{\beta}}+C C_{\bar{\alpha} \bar{\beta}}\right)+e\left((-1)^{\beta} V_{g}+\frac{V}{2}\right)\left(C_{\Sigma \bar{\alpha}} C_{g \alpha}+C C_{g \bar{\alpha}}\right)\right] \\
\Delta \overleftarrow{K}_{\alpha \beta}=\frac{1}{C_{\Delta}^{2}}[ & -e^{2}\left(C_{\Sigma \bar{\alpha}}\left((-1)^{\beta} n_{\alpha}+\frac{1}{2}\right)+C(-1)^{\beta} n_{\bar{\alpha}}\right)- \\
& \left.-e V\left(C_{\Sigma \bar{\alpha}} C_{\alpha \bar{\beta}}+C C_{\bar{\alpha} \bar{\beta}}\right)-e\left((-1)^{\beta} V_{g}+\frac{V}{2}\right)\left(C_{\Sigma \bar{\alpha}} C_{g \alpha}+C C_{g \bar{\alpha}}\right)\right] .
\end{aligned}
$$

For the tunneling between grains we have

$$
\begin{array}{r}
\Delta K_{\alpha \bar{\alpha}}=\frac{1}{C_{\Delta}^{2}}\left[-e^{2}\left(-C_{\Sigma \bar{\alpha}}\left(n_{\alpha}-\frac{1}{2}\right)+C_{\Sigma \alpha}\left(n_{\bar{\alpha}}+\frac{1}{2}\right)+C\left(n_{\alpha}-n_{\bar{\alpha}}-1\right)\right)+\right. \\
-e(-1)^{\alpha} V\left(C_{\alpha \alpha} C_{\bar{\alpha} \bar{\alpha}}-C_{\alpha \bar{\alpha}} C_{\bar{\alpha} \alpha}+C_{g \alpha} \frac{C_{\bar{\alpha} \bar{\alpha}}-C_{\bar{\alpha} \alpha}}{2}+C_{g \bar{\alpha}} \frac{C_{\alpha \alpha}-C_{\alpha \bar{\alpha}}}{2}\right)+ \\
\left.+e V_{g}\left(C_{g \alpha}\left(C_{\bar{\alpha} \bar{\alpha}}+C_{\bar{\alpha} \alpha}\right)-C_{g \bar{\alpha}}\left(C_{\alpha \alpha}+C_{\alpha \bar{\alpha}}\right)\right)\right]
\end{array}
$$

Here we defined $C_{\Sigma 1}=C_{11}+C_{12}+C_{g 1}+C, C_{\Sigma 2}=C_{21}+C_{22}+C_{g 2}+C$, and $C_{\Delta}^{2}=C_{\Sigma 1} C_{\Sigma 2}-C^{2}$. We also defined $\bar{\alpha}=2$ if $\alpha=1$ and $\bar{\alpha}=1$ if $\alpha=2$; the same definition for $\bar{\beta}$.

Equations (2.1) and (2.2) are highly symmetric - see Appendix A.

\section{QUASIPERIODICITY}

The position of Coulomb staircases in a $\frac{\mathrm{dI}}{\mathrm{dV}}\left(V, V_{g}\right)$ plot is determined by the condition that the electrostatic energy decrement on tunneling through some barrier crosses zero:

$\Delta \vec{K}_{\alpha \beta}\left(n_{1}, n_{2}, V, V_{g}\right)=0$.

From (2.1) and (2.2) we have:

$$
\begin{gathered}
V\left(C_{12}+\frac{C}{C_{\Sigma 2}} C_{22}\right)-\left(V_{g}-\frac{V}{2}\right)\left(C_{g 1}+\frac{C}{C_{\Sigma 2}} C_{g 2}\right)=e\left(n_{1}+\frac{C}{C_{\Sigma 2}} n_{2} \pm \frac{1}{2}\right), \\
-V\left(C_{11}+\frac{C}{C_{\Sigma 2}} C_{21}\right)-\left(V_{g}+\frac{V}{2}\right)\left(C_{g 1}+\frac{C}{C_{\Sigma 2}} C_{g 2}\right)=e\left(n_{1}+\frac{C}{C_{\Sigma 2}} n_{2} \pm \frac{1}{2}\right), \\
V\left(\frac{C}{C_{\Sigma 1}} C_{12}+C_{22}\right)-\left(V_{g}-\frac{V}{2}\right)\left(\frac{C}{C_{\Sigma 1}} C_{g 1}+C_{g 2}\right)=e\left(\frac{C}{C_{\Sigma 1}} n_{1}+n_{2} \pm \frac{1}{2}\right), \\
-V\left(\frac{C}{C_{\Sigma 1}} C_{11}+C_{21}\right)-\left(V_{g}+\frac{V}{2}\right)\left(\frac{C}{C_{\Sigma 1}} C_{g 1}+C_{g 2}\right)=e\left(\frac{C}{C_{\Sigma 1}} n_{1}+n_{2} \pm \frac{1}{2}\right), \\
V\left(C_{11} C_{22}-C_{12} C_{21}+C_{g 1} \frac{C_{22}-C_{21}}{2}+C_{g 2} \frac{C_{11}-C_{12}}{2}\right)+ \\
+V_{g}\left(C_{g 1}\left(C_{22}+C_{21}\right)-C_{g 2}\left(C_{11}+C_{12}\right)\right)= \\
=e\left(-\left(C_{\Sigma 2}-C\right) n_{1}+\left(C_{\Sigma 1}-C\right) n_{2} \pm\left(\frac{1}{2} C_{\Sigma 1}+\frac{1}{2} C_{\Sigma 2}-C\right)\right) .
\end{gathered}
$$


Because of time-reversal symmetry between tunneling jumps $\alpha \rightarrow \beta$ and $\beta \rightarrow \alpha$, the conditions $\Delta \vec{K}_{\alpha \beta}\left(n_{\alpha}\right)=0$ and $\Delta \overleftarrow{K}_{\alpha \beta}\left(n_{\alpha}+1\right)=0$ impose the same relationship on $V, V_{g}$, and only 5 distinct sets of pecularities are defined by (3.1).

We can draw some important conclusions from (3.1):

a. All Coulomb pecularities form straight lines in a $\frac{\mathrm{dI}}{\mathrm{dV}}\left(V, V_{g}\right)$ plot. Indeed, any equation in (3.1) has a linear form

$$
A_{\alpha \beta} V+B_{\alpha \beta} V_{g}=C_{\alpha \beta} n_{1}+D_{\alpha \beta} n_{2}+\text { const. }
$$

b. Each tunneling barrier defines a set of pecularities which are parallel to each other, i. e. a double dot system has maximum five distinct slopes in the $\frac{\mathrm{dI}}{\mathrm{dV}}$ pattern.

c. There is no more periodicity with respect to a gate voltage, like in a single-dot system. Nevertheless, the whole $\frac{\mathrm{dI}}{\mathrm{dV}}$ pattern is quasiperiodic, because all staircases coming from the first grain have offsets $n_{1}+\left(C / C_{\Sigma 2}\right) n_{2}+\cdots$, from the second one - $\left(C / C_{\Sigma 1}\right) n_{1}+n_{2}+\ldots$, and from intergrain tunneling $-\left(C_{\Sigma 2}-C\right) n_{1}-\left(C_{\Sigma 1}-C\right) n_{2}+\cdots$.

\section{MASTER EQUATION FOR EVOLUTION}

If both grains have a continuos spectrum, then for tunneling rates $\Gamma_{\alpha, \beta}\left(n_{1}, n_{2}\right)$ through the lead-grain gaps we have:

$$
\begin{aligned}
& \vec{\Gamma}_{\alpha \beta}=\frac{\frac{1}{e} I_{\alpha \beta}\left(\frac{\Delta \vec{K}_{\alpha \beta}}{e}\right)}{1-\exp \left(-\frac{\Delta \vec{K}_{\alpha \beta}}{k T}\right)}, \\
& \overleftarrow{\Gamma}_{\alpha \beta}=\frac{\frac{1}{e} I_{\alpha \beta}\left(\frac{\Delta \overleftarrow{K}_{\alpha \beta}}{e}\right)}{1-\exp \left(-\frac{\Delta \overleftarrow{K}_{\alpha \beta}}{k T}\right)}, \alpha, \beta=1,2 ;
\end{aligned}
$$

and for intergrain tunneling rates:

$$
\Gamma_{\alpha \bar{\alpha}}=\frac{\frac{1}{e} I_{\alpha \bar{\alpha}}\left(\frac{\Delta K_{\alpha \bar{\alpha}}}{e}\right)}{1-\exp \left(-\frac{\Delta K_{\alpha \bar{\alpha}}}{k T}\right)}, \alpha=1,2,
$$

where $I_{\alpha \beta}(V)$ is the current-voltage characteristics of the corresponding tunneling barrier. Assuming Ohms law, we put $I_{\alpha \beta}(V)=G_{\alpha \beta} V$, but all formulas can be easily generalized for the case of a discrete energy spectrum in the grains.

The master equation for the double dot system is

$$
\begin{aligned}
& \dot{p}_{n 1, n 2}=-\left(\vec{\Gamma}_{11}\left(n_{1}, n_{2}\right)+\overleftarrow{\Gamma}_{11}\left(n_{1}, n_{2}\right)+\vec{\Gamma}_{12}\left(n_{1}, n_{2}\right)+\overleftarrow{\Gamma}_{12}\left(n_{1}, n_{2}\right)+\right. \\
& \vec{\Gamma}_{21}\left(n_{1}, n_{2}\right)+\overleftarrow{\Gamma}_{21}\left(n_{1}, n_{2}\right)+\vec{\Gamma}_{22}\left(n_{1}, n_{2}\right)+\overleftarrow{\Gamma}_{22}\left(n_{1}, n_{2}\right)+ \\
&\left.+\Gamma_{12}\left(n_{1}, n_{2}\right)+\Gamma_{21}\left(n_{1}, n_{2}\right)\right) p_{n_{1}, n_{2}}+ \\
&+\left(\vec{\Gamma}_{11}\left(n_{1}-1, n_{2}\right)+\overleftarrow{\Gamma}_{12}\left(n_{1}-1, n_{2}\right)\right) p_{n_{1}-1, n_{2}}+ \\
&+\left(\vec{\Gamma}_{21}\left(n_{1}, n_{2}-1\right)+\overleftarrow{\Gamma}_{22}\left(n_{1}, n_{2}-1\right)\right) p_{n_{1}, n_{2}-1}+ \\
&+\left(\overleftarrow{\Gamma}_{11}\left(n_{1}+1, n_{2}\right)+\vec{\Gamma}_{12}\left(n_{1}+1, n_{2}\right)\right) p_{n_{1}+1, n_{2}}+ \\
&+\left(\overleftarrow{\Gamma}_{21}\left(n_{1}, n_{2}+1\right)+\vec{\Gamma}_{22}\left(n_{1}, n_{2}+1\right)\right) p_{n_{1}, n_{2}+1}+ \\
&+\Gamma_{21}\left(n_{1}-1, n_{2}+1\right) p_{n_{1}-1, n_{2}+1}+\Gamma_{12}\left(n_{1}+1, n_{2}-1\right) p_{n_{1}+1, n_{2}-1}
\end{aligned}
$$

And the current is given by

$$
I=e \sum_{n 1, n 2=-\infty}^{+\infty}\left(\vec{\Gamma}_{11}\left(n_{1}, n_{2}\right)-\overleftarrow{\Gamma}_{11}\left(n_{1}, n_{2}\right)+\vec{\Gamma}_{21}\left(n_{1}, n_{2}\right)-\overleftarrow{\Gamma}_{21}\left(n_{1}, n_{2}\right)\right) p_{n_{1}, n_{2}}
$$




\section{NUMERIC SOLUTION OF A DOUBLE DOT SET}

Master equation (4.3) can be solved numerically in a straightforward manner: for any given $\left(V, V_{g}\right)$ one should trace the evolution of $p_{n, m}$ until an equilibrium distribution is reached (see Appendix B for the details of the numeric procedure), the current is then given by (4.4). This way the current can be computed for an arbitrary double grain system.

To test the program, we solved a general double dot system and compared the program output with the result of a Monte-Carlo simulation obtained with a commercial single electron circuit simulation software - SIMON 2.0 [17]. As one can see, all Coulomb peculiarities have identical positions in both Fig. 2a and Fig. 2pb.

\section{DOUBLE DOT SYSTEM WITH STRONGLY ASYMMETRIC TUNNELING BARRIERS}

Fig. 2 illustrates the common rule: in a multigrain system any tunneling gap gives a set of peculiarities on $\frac{\mathrm{dI}}{\mathrm{dV}}\left(V, V_{g}\right)$ plot with one and just one unique slope. Equation set (3.1), however, only specifies possible staircase positions, not amplitudes. The amplitudes depend on probability distribution $p_{n_{1}, n_{2}}$ as defined by (4.3).

For example, lets consider the case of negative bias voltage $V$ with electrons flowing in Source-to-Drain direction. Whenever the small variation of $V$ and/or $V_{g}$ makes, say, $\Delta \vec{K}_{11}(n, m)$ positive, electron from Fermi level in a Source lead can tunnel into a Fermi level in the first grain. One additional intermediate charge state $\{n+1, m\}$ becomes accessible, resulting in one extra term in current sum (4.4) due to to $\{n, m\} \rightarrow\{n+1, m\}$ transitions. The resulting current increment, however, is proportional to the probability of a system to be found in a $\{n, m\}$ state multiplied by $\{n, m\} \rightarrow\{n+1, m\}$ tunneling rate: $\vec{\Gamma}_{11}(n, m) p_{n, m}$. If $p_{n, m}$ is small, i.e. the $\{n, m\}$ state is statistically unfeasible, then a newly opened channel does not contribute much the total current, and the corresponding staircase is suppressed.

For clarification, we can refer to a single grain SET. In a symmetric case $\left(G_{1}=G_{2}\right)$ all charge states, accessible from the energy considerations, have comparable feasibility: $p_{n_{\max }} \sim p_{n_{\max }-1} \sim \ldots \sim p_{-n_{\max }}$. On the contrary, in the asymmetric case $G_{1} \gg G_{2}$ the grain is almost in equilibrium with the low resistance electrode. In a rare event of tunneling through the low-conductive gap $G_{2}$ equilibrium is violated, but almost immediately restored by tunneling through a high-conductive barrier $G_{1}$. Two successive tunneling events through $G_{2}$ not interleaved by tunneling through $G_{1}$ are even less feasible. As a result, the charge fluctuations are strongly suppressed and in the $G_{2} \rightarrow 0$ limit the probability distribution reduces to a single charge state $n_{\max }: p_{n}=\delta_{n, n_{\max }}$.

Whenever a bias or gate increment makes tunneling through the $G_{1}$ gap into the $n+1$ state possible, an equilibrium grain charge switches to $e(n+1)$, and the current is incremented stepwise. On the contrary, when the voltage variation opens a new tunneling channel through the $G_{2}$ gap, the equilibrium charge remains the same, and no current step takes place. While a symmetric SET has two distinct staircase families, the first one defined by $K_{1}\left(n, V, V_{g}\right)=0$ and the second by $K_{2}\left(n, V, V_{g}\right)=0$, in an asymmetric system one of them is suppressed.

Exactly the same kinetic effect takes place for a double grain (multigrain) SET: if tunneling gaps have strongly different conductances $G_{\alpha \beta}$, then the vast majority of accessible charge states becomes unfeasible, and for any given pair $\left(V, V_{g}\right)$ just one charge state $\left\{n_{1}\left(V, V_{g}\right), n_{2}\left(V, V_{g}\right)\right\}$ dominates. If only $V$ and/or $V_{g}$ variation changes the dominant charge state, a prominent staircase appears. For any grain $\alpha$, the grain charge is defined by the most conducting tunneling gap $\beta_{\max }$, and the charge state changes when $K_{\alpha \beta_{\max }}\left(n_{1}, n_{2}, V, V_{g}\right)$ crosses zero. All other staircases, corresponding $K_{\alpha, \beta \neq \beta \max }$ are supressed.

To summarize, in a multigrain system each tunneling gap defines one staircase family with a distinct slope in the $\left(V, V_{g}\right)$ plane. But in a strongly asymmetric system only one staircase family per every grain survives - the one corresponding to the most conductive link. Although in the double grain system there may be up to five distinct staircase families, in the asymmetric case only two will stay - the first one corresponding to the $n_{1}$ increments and the second one to $n_{2}$ increments. Three-grain system will have at least three staircase families and so on. And vice versa - a system with $n$ distinct staircase slopes has no more than $n$ grains.

This qualitative consideration is confirmed by the numeric result presented in Fig. 3 .

\section{A SYSTEM WITH TWO STAIRCASE FAMILIES: POSSIBLE TOPOLOGIES}

As it was discussed above, a system with two staircase families may have maximum two grains. Each grain must have one strong tunneling link with conductance much higher than the other tunneling gaps adjacent to this grain. This allows us to list all possible topologies directly. We note first that each grain has three links, and any one could 
be dominant. Therefore, for two grains we have $3 \times 3$ different possibilities, all shown in Fig 1 . (note that for generality we assume that the grains have different size). Variants $\mathbf{a}, \mathbf{c}, \mathbf{e}$, and $\mathbf{g}$ are obviously symmetric to $\mathbf{b}, \mathbf{d}, \mathbf{f}$, and $\mathbf{h}$ respectively. Variant $\mathbf{i}$ has no more than two staircase families if only one of the grain-electrode gaps has much higher conductance than the others. In this case the system reduces either to $\mathbf{d}$ or $\mathbf{f}$. To summarize, we have four distinct cases: $\mathbf{b}, \mathbf{d}, \mathbf{f}$, and $\mathbf{h}$; we shall consider each one in more detail below 7 .

Grains are linked to different electrodes - the case in Fig. 斯. Two visible staircase families are those coming from the gaps $G_{11}$ and $G_{22}$. The $\mathrm{d} I / \mathrm{d} V\left(V, V_{g}\right)$ plot looks slightly different depending on which of the remaining tunneling gaps - $G_{12}, G_{21}$ or $G$ has the highest conductivity. The current either flows mainly through the big grain $\left(G_{21}>\max \left(\mathrm{G}, \mathrm{G}_{12}\right)\right.$ - the numeric result is shown in Fig. Eb $\left.1 \mathrm{~b}\right)$, through the small one $\left(G_{12}>\max \left(\mathrm{G}, \mathrm{G}_{21}\right)-\right.$ see Fig. 5b2), or two grains connected in series $\left(G>\max \left(\mathrm{G}_{21}, \mathrm{G}_{12}\right)\right.$ - presented in Fig. 3b b . Note that in all cases the Coulomb staircases have exactly the same position in the $\left(V, V_{g}\right)$ plane, but the staircase amplitudes (and even the signs) are different. The regions of the Coulomb blockade are also different. In the general case of $G_{12} \sim G_{21} \sim G$ the Coulomb blockade is lifted as soon as at least one of these current channels opens for transport.

A big grain is linked to a small one, which is linked to an electrode - the case in Fig. G $\mathrm{d}$. The current either goes through the big grain $\left(G_{22}>G_{12}\right)$ or bypasses it $\left(G_{22}<G_{12}\right)$. The corresponding $\mathrm{d} I / \mathrm{d} V$ plots are shown in Fig. $5 \mathrm{~d} \mathrm{~d} 1$ and Fig. . $\mathrm{d} 1$ respectively.

A small grain is linked to a big one, which is linked to an electrode - the case in Fig. Q1f. Two possible topologies complementary to d1 and $\mathrm{d} 2$ are presented in Fig. 5f 1 and Fig. Ff 2 together with $\mathrm{d} I / \mathrm{d} V$ plots.

The last possibility - both grains are linked to the same electrode - is shown in Fig. 蛙. If the current goes mainly through the small grain, we have the $\mathrm{d} I / \mathrm{d} V$ pattern presented in Fig. E. $\mathrm{h} 1$, otherwise - the one in Fig $5 \mathrm{~h} 2$.

In any case $\mathrm{d} I / \mathrm{d} V$ pattern has a simple structure: there are big areas shaded by parallel and equdistant spectral lines (one is shown by dotted line in Fig. 5h1). The reason for this highly regular structure is quite obvious for topologies in b1, b2, h1, h2 - a single grain SET coupled to a Single Electron Box (SEB). Indeed, until SEB charge $Q_{\mathrm{SEB}}$ remains constant, the SEB affects SET in essentially the same way as a constant background charge (of course, there is also some capacitance renormalisation). So all SET staircases are periodic with $\left(V, V_{g}\right)$. Whenever $Q_{\mathrm{SEB}}$ changes, the SET feels a different "background charge" and the phase of its Coulomb blockade oscillations is shifted. Remarkably, this high regularity survives even if two grains are connected in series, as in $\mathrm{d} 1$ and $\mathrm{f} 1$.

\section{COMPARISON WITH EXPERIMENT}

The analysis presented in this paper was to a large extent motivated by our recent experiments with nanoconstrictions in Quench-Condenced (QC) films [9, 10]. We have developed a method to define constrictions with width and length of about $5 \mathrm{~nm}$, bridged to two macroscopic electrodes. At small thiknesses QC films are granular [18], so there are typically a few metallic grains separated by tunneling gaps within such a constriction - see Fig. 6 for the sample geometry. By additional in situ film depositions we merged some clusters together, finally reducing the sample topology to a single grain SET. The only opportunity to follow the sample topology was the analysis of the sample's $\mathrm{dI} / \mathrm{dV}\left(\mathrm{V}, \mathrm{V}_{\mathrm{g}}\right)$ pattern. We used the technique presented here to determine the number of grains in the constriction, to reconstruct the sample topology, and to extract all grain capacitances. The fast computation time (compared to the Monte-Carlo method) makes this procedure practical.

The strong exponential dependence of the tunneling conductance on the gap width greatly simplifies the spectral pattern. Indeed, we can assume that the tunneling gaps between the grains are randomly and uniformly distributed from $1 \AA$ to $10 \AA$. Then the tunneling resistances are randomly and uniformly distributed in logarithmic scale from $10 \mathrm{k} \Omega$ and up to $1 \mathrm{G} \Omega$. This virtually eliminates the kinetic charge fluctuations, and washes out all but one staircase family per grain, i. e. every staircase family identifies the unique grain.

A typical experimental data are shown in Fig. 7 together with a numeric fit. The reconstructed sample topology is presented in Fig. 月l : two grains, with charging energies $e^{2} / 2 C_{\Sigma}$ about 9 and $34 \mathrm{meV}$, are connected in series; and there is a small single electron box with charging energy $\sim 52 \mathrm{meV}$ nearby.

Note that the inter-grain capacitance $C$ is always small compared to coupling to the leads or the gate electrode (see caption to Fig. 7). This is because an inter-grain interaction is effectively screened by the common gate electrode. For

\footnotetext{
${ }^{1}$ Note that it is natural to assume in $\mathbf{b}, \mathbf{d}$, and $\mathbf{f}$ cases that a smaller grain is closer to the left electrode, and the bigger grain - to the right one. That's why all numeric results presented below were computed for a system with $C_{11}>C_{12}$ and $C_{21}<C_{22}$. We put this constraint to make the staircase slopes more realistic, it does not affect the cardinal pattern of $\mathrm{dI} / \mathrm{dV}$ plot.
} 
the sample in Fig. 月 the gate oxide layer was about $3 \mathrm{~nm}$ thick, so for two grains bigger than $\sim 3 \mathrm{~nm}$ we always have $C<C_{g}$. Moreover, any two grains placed more than a few nanometers away virtually do not interfere. This allows to apply our analysis of a double-grain system recursively, and to identify all grains in a multi-grain system one by one.

Whenever an additional deposition merges two grains, one staircase family disappears. This evolution eventually leads to a single grain SET, and after a few additional depositions, to a single tunneling gap separating two macroscopic electrodes 10].

Intriguingly, for samples with very high charging energies $(\gtrsim 100 \mathrm{meV})$, we have always observed some non-orthodox spectral lines like the ones marked with $\gamma$ in Fig. 8. All lines $\gamma$ have the same slope, different from the slope $\beta$ of the main Coulomb staircases. As it was shown previously, a system with two distinct slopes on the $\mathrm{d} I / \mathrm{d} V$ pattern has no more than two grains involved in current trunsport. Would the lines $\gamma$ be due to recharging of some other grains, they all must come from recharging of one and the same grain. In this case they must be equidistant, which obviously does not take place.

\section{CONCLUSION}

We proved the following statements for a multigrain system in the framework of the ortodox model:

1. All Coulomb peculiarities in differential conductance form straight lines on the gate-bias plane.

2. Each tunneling gap defines a set of staircases, which are parallel to each other. In an asymmetric system with strongly different tunneling resistances only one distinct staircase family per every grain survives - the one corresponding to the most conductive link; all other staircases are suppressed.

3. There may be a maximum of $n$ grains in a system with $n$ staircase slopes.

We presented the exact solution for an arbitrarily connected double-grain system. We demonstrated that although the zero-bias conductance is stochastic [19], the large-scale spectroscopy pattern $\mathrm{d} I / \mathrm{d} V\left(V, V_{g}\right)$ (differential conductance as a function of bias and gate voltages) is regular - all Coulomb staircases are quasiperiodic.

We listed all possible topologies for a system with two different staircase slopes and presented the corresponding spectral patterns.

Finally, we proved the following criterion:

If there are only two slopes $\alpha$ and $\beta$ for all spectral lines on spectroscopy pattern, and the lines with the slope $\beta$ are not equidistant within the regions between two adjacent lines alpha, then they can not be attributed to the presence of one or more additional grains in the samplef.

To conclude, we have analysed a multigrain system with a shared gate and found that the sample topology can be unambiguously deduced from $\mathrm{d} I / \mathrm{d} V\left(V, V_{g}\right)$ pattern. We demonstrated how to distinguish peculiarities in tunneling spectra of single-grain samples from possible disturbance caused by some extra grains. Presented analysis can be applied for numerous systems, where the experimentalist is not able to image the sample directly.

\section{ACKNOWLEDGMENTS}

We are grateful to T. Claeson and A.Ya. Tzalenchuk for illuminating discussions and critical readings of the manuscript. The work was supported by the Royal Swedish Academy of Sciences, through its Nobel Institute, by Swedish Foundation of Strategic Research, and by European Union under IST-FET NANOMOL program.

\section{APPENDIX A: SYMMETRY CONSIDERATIONS}

Equations (2.1) and (2.2) are invariant with respect to the following symmetry transformations.

\footnotetext{
${ }^{2}$ This criterion can be generalized in a straightforward manner to the case of $n$ slopes on $\mathrm{d} I / \mathrm{d} V$ pattern, but this situation seems to be of low practical importance.
} 
a. We can swap indices marking the grain numbers, i. e. we can redeclare grain no 1 as 2 and vice versa. So as a result of simultaneous replacement

$n_{1} \leftrightarrows n_{2}, C_{11} \leftrightarrows C_{21}, C_{12} \leftrightarrows C_{22}, C_{g 1} \leftrightarrows C_{g 2}$, and $\mathrm{C}_{\Sigma 1} \leftrightarrows \mathrm{C}_{\Sigma 2}$

we will have the following transformations:

$\Delta \vec{K}_{1 \beta}\left(n_{1}, n_{2}\right) \leftrightarrows \Delta \vec{K}_{2 \beta}\left(n_{2}, n_{1}\right), \Delta \overleftarrow{K}_{1 \beta}\left(n_{1}, n_{2}\right) \leftrightarrows \Delta \overleftarrow{K}_{2 \beta}\left(n_{2}, n_{1}\right)$, and

$\Delta \mathrm{K}_{12}\left(\mathrm{n}_{1}, \mathrm{n}_{2}\right) \leftrightarrows \Delta \mathrm{K}_{21}\left(\mathrm{n}_{2}, \mathrm{n}_{1}\right)$

b. Left/right symmetry: we can flip the whole network horizontally with simultaneous inversion of the bias voltage, which is equivalent to the following substitution:

$C_{11} \leftrightarrows C_{12}, C_{21} \leftrightarrows C_{22}, V \leftrightarrows-V$

As a result, the tunneling jump from, say, the left tunneling barrier will be transformed into a jump through the right barrier in the opposite direction, so as

$\Delta \vec{K}_{\alpha 1}\left(V, V_{g}\right) \leftrightarrows \Delta \overleftarrow{K}_{\alpha 2}\left(-V, V_{g}\right), \Delta \overleftarrow{K}_{\alpha 1}\left(V, V_{g}\right) \leftrightarrows \Delta \vec{K}_{\alpha 2}\left(-V, V_{g}\right)$

Energy decrements for intergrain tunneling $\Delta K_{12}$ and $\Delta K_{21}$ are not affected by this transformation and they will convert to themselves.

c. Time inversion symmetry: if for any tunneling event from state $n_{1}, n_{2}$ to state $n_{1}^{\prime}, n_{2}^{\prime}$ the energy decrement is $\Delta K$, then the inverse tunneling process from $n_{1}^{\prime}, n_{2}^{\prime}$ to $n_{1}, n_{2}$ must have the opposite energy decrement $-\Delta K$, and so $\Delta \vec{K}_{11}\left(n_{1}, n_{2}\right)=-\Delta \overleftarrow{K}_{11}\left(n_{1}+1, n_{2}\right), \Delta \vec{K}_{12}\left(n_{1}, n_{2}\right)=-\Delta \overleftarrow{K}_{12}\left(n_{1}-1, n_{2}\right)$

$\Delta \vec{K}_{21}\left(n_{1}, n_{2}\right)=-\Delta \overleftarrow{K}_{21}\left(n_{1}, n_{2}+1\right), \Delta \vec{K}_{22}\left(n_{1}, n_{2}\right)=-\Delta \overleftarrow{K}_{22}\left(n_{1}, n_{2}-1\right)$ and $\Delta K_{12}\left(n_{1}, n_{2}\right)=-\Delta K_{21}\left(n_{1}-1, n_{2}+1\right)$.

Any of a-c symmetries reduces the number of independent formulas in (2.1) by a factor of 2 , so there is really just one independent equation in (2.1).

There is yet another symmetry in (2.1) and (2.2):

d. All energy decrements change the sign and the tunneling direction on simultaneous inversion of all voltages and charges, i. e.

$\Delta \vec{K}_{\alpha \beta}\left(n_{1}, n_{2}, V, V_{g}\right)=-\Delta \overleftarrow{K}_{\alpha \beta}\left(-n_{1},-n_{2},-V,-V_{g}\right)$, and

$\Delta \overleftarrow{K}_{\alpha \beta}\left(n_{1}, n_{2}, V, V_{g}\right)=-\Delta \vec{K}_{\alpha \beta}\left(-n_{1},-n_{2},-V,-V_{g}\right)$

If we apply this transformation to the expression for current (4.4) then the current will change sign:

$$
I\left(V, V_{g}\right)=-I\left(-V,-V_{g}\right), \text { and } \frac{\mathrm{dI}}{\mathrm{dV}}\left(V, V_{g}\right)=\frac{\mathrm{dI}}{\mathrm{dV}} I\left(-V,-V_{g}\right)
$$

So the $I\left(V, V_{g}\right)$ plot is antisymmetric, and the $\frac{\mathrm{dI}}{\mathrm{dV}}\left(V, V_{g}\right)$ plot is symmetric. In the presence of a background charge, the relative phase of Coulomb oscillations on the first and the second grain will be shifted and this symmetry will be violated.

If we complete transformation $\mathbf{d}$ with the inversion of the electron charge $e \rightarrow-e$ then the current will not change. This could be reformulated as follows: the current through the system does not depend on the sign of charge carriers, i. e. whether the current transport is carried by electrons or holes.

\section{APPENDIX B: NUMERICAL RECIPIES}

The master equation (4.3) is a system of linear differential equations. If we present the probability distribution $p_{n_{1}, n_{2}}$ as a vector $\vec{p}=\left|p_{j}\right\rangle$ where $j$ is an index running over all $\left(n_{1}, n_{2}\right)$ pairs, then we can rewrite (4.3) as

$$
\dot{\vec{p}}=-\widehat{\Gamma} \vec{p}
$$

The formal solution of $(12.1)$ is

$$
\begin{aligned}
\vec{p}(t) & =e^{-t \widehat{\Gamma}} \vec{p}(0)=\left(\widehat{\mathrm{E}}-\mathrm{t} \widehat{\Gamma}+\frac{\mathrm{t}^{2}}{2} \widehat{\Gamma}^{2}-\frac{\mathrm{t}^{3}}{6} \widehat{\Gamma}^{3}+\frac{\mathrm{t}^{4}}{24} \widehat{\Gamma}^{4}+\cdots\right) \\
& =(\widehat{\mathrm{E}}-\mathrm{t} \widehat{\Gamma})\left(\widehat{\mathrm{E}}-\frac{\mathrm{t}}{2} \widehat{\Gamma}\right)\left(\widehat{\mathrm{E}}-\frac{\mathrm{t}}{3} \widehat{\Gamma}\right)\left(\widehat{\mathrm{E}}-\frac{\mathrm{t}}{4} \widehat{\Gamma}\right) \overrightarrow{\mathrm{p}}(0)+\mathrm{o}\left(\mathrm{t}^{4}\right),
\end{aligned}
$$

where $\widehat{\mathrm{E}}$ is the unity matrix.

As one can see from (12.2), the standard fourth-order Runge-Kutta method reduces in our case to four recursive calls to a first-order Eiler's extrapolation formula with steps $\frac{t}{4}, \frac{t}{3}, \frac{t}{2}, t$. 
To minimise execution time, it is better to use an adaptive step $t$. Ideally, it should be inversely proportional to the maximum eigenvalue of the evolution matrix $\widehat{\Gamma} \lambda_{\max }$. Practically, it is good enough to replace an unknown $\lambda_{\max }$ with the sum of all eigenvalues $\sum \lambda_{j}=\operatorname{tr}(\widehat{\Gamma})$, where the trace of the evolution matrix can be found from $(4.3)$ and equals

$$
\operatorname{tr}(\widehat{\Gamma})=\sum_{n 1, n 2=-\infty}^{\infty}\left(\sum_{\alpha, \beta=1,2}\left(\vec{\Gamma}_{\alpha \beta}\left(n_{1}, n_{2}\right)+\overleftarrow{\Gamma}_{\alpha \beta}\left(n_{1}, n_{2}\right)\right)+\sum_{\alpha=1,2} \Gamma_{\alpha \bar{\alpha}}\left(n_{1}, n_{2}\right)\right)
$$

[1] D.L. Klein, R. Roth, A.K.L. Lim, A.P. Alivisatos, P.L. McEuen, Nature 389, 699 (1997).

[2] S.H.M. Persson, L.G.M. Olofsson, L.K. Gunnarsson, Appl. Phys. Lett. 74, 2546 (1999).

[3] E.S. Soldatov, V.V. Khanin, A.S. Trifonov, D.E. Presnov, S.A. Yakovenko, G.B. Khomutov, G.P. Gubin, V.V. Kolesov JETP. Lett. 64, 556 (1996).

[4] H.Park, J.Park, A.K.L. Lim, E.K. Anderson, A.P. Alivisatos, P.L. McEuen, Nature 407, 57 (2000).

[5] D.C. Ralph, C.T. Black, M. Tinkham, Phys. Rev. Lett. 74, 3241 (1995).

[6] D.C. Ralph, C.T. Black, M. Tinkham, Phys. Rev. Lett. 78, 4087 (1997).

[7] S. Gueron, M.M. Deshmukh, E.B. Myers, D.C. Ralph, Phys. Rev. Lett. 83, 4148 (1999).

[8] D. Davidović, M. Tinkham, Phys. Rev. Lett. 83, 1644 (1999).

[9] S.E. Kubatkin, A.V. Danilov, A.L. Bogdanov, H. Olin, T. Claeson, Appl. Phys. Lett. 73, 3604 (1998).

[10] S.E. Kubatkin, A.V. Danilov, H. Olin, T. Claeson, J. Low Temp. Phys. 118, 307 (2000).

[11] D.V. Averin and K.K. Likharev, in Mesoscopic Phenomena in Solids, (North-Holland, New York, 1991), Chap. 6.

[12] I.M. Ruzin, V. Chandrasekhar, E.I. Levin, L.I. Glasman, Phys. Rev. B 45, 13469 (1992).

[13] L.P. Kouwenhoven, T.H. Oosterkamp, M.W.S. Danoesastro, M. Eto, D.G. Austing, T. Honda, S. Tarucha, Science 278, 1788 (1997).

[14] A conclusive argument is observation of the Zeeman shift for quantized energy levels in a magnetic field [6], but to observe this effect in a magnetic field below $10 \mathrm{~T}$ one should lower the temperature to a dilution refrigerator range.

[15] N.F. Schwabe, A.N. Cleland, M.C. Cross, M.L. Roukes, Phys. Rev. B 52, 12911 (1995).

[16] L.Y. Gorelik, A. Isacsson, M.V. Voinova, B. Kasemo, R.I. Shekhter, M. Jonson, Phys. Rev. Lett. 80, 4526 (1998).

[17] URL: http://home1.gte.net/kittypaw/simon.htm.

[18] K.L. Ekinchi, J.M. Valles, Jr., Phys. Rev. B 58, 7347 (1998); K.L. Ekinchi, J.M. Valles, Jr., Phys. Rev. Lett. 82, 1518 (1999).

[19] A system of two grains connected in series was studied in [12]. It was shown, that at temperatures below the smallest charging energy, the zero bias conductance, as a function of the gate voltage, is a set of randomly placed peaks with random amplitudes. 

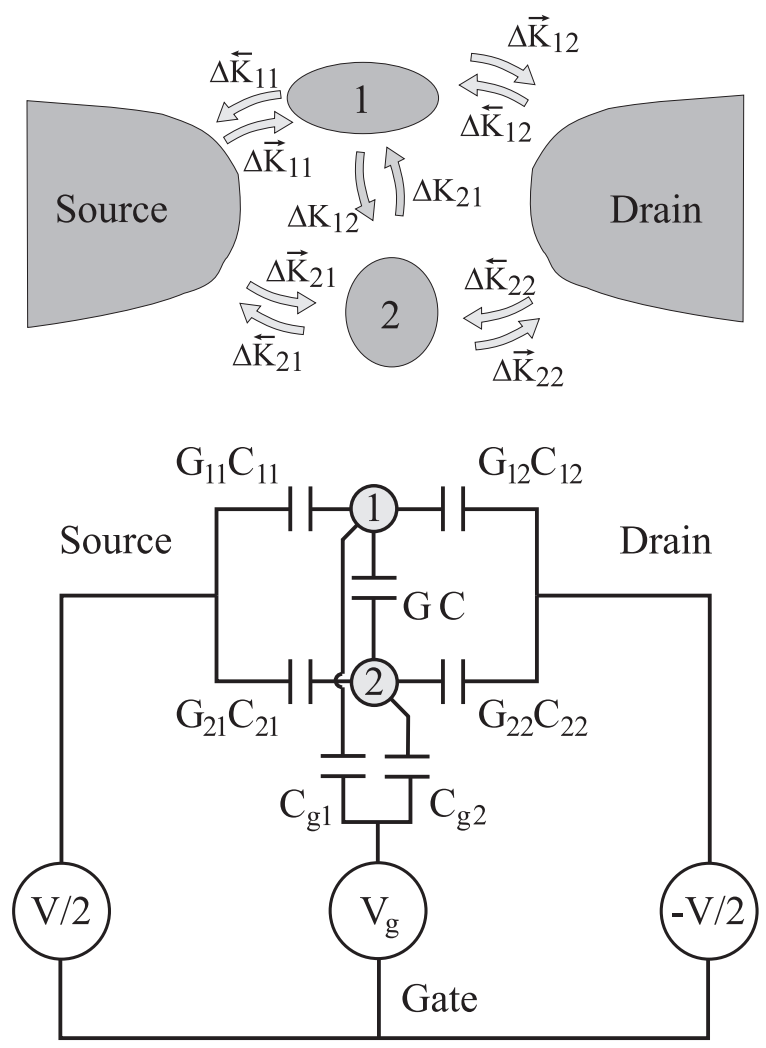

FIG. 1. Top: system topology and energy decrements on tunneling jumps; Bottom: equivalent schematics.
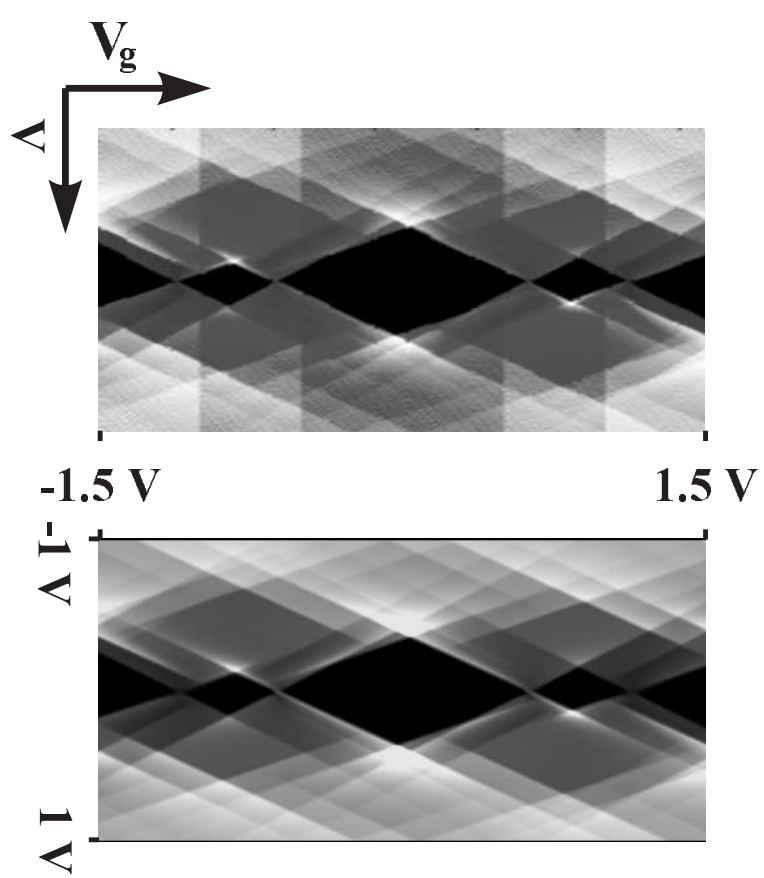

FIG. 2. General case of a double dot SET: differential conductance plot as a function of bias and gate voltages. a.- The result of Monte-Carlo simulation. b.- Direct numeric integration of master equation. System parameters: $C_{11}=16 \times 10^{-20} \mathrm{~F}$, $C_{12}=8 \times 10^{-20} \mathrm{~F}, C_{21}=9 \times 10^{-20} \mathrm{~F}, C_{22}=13 \times 10^{-20} \mathrm{~F}, C_{g 1}=8 \times 10^{-20} \mathrm{~F}, C_{g 2}=11 \times 10^{-20} \mathrm{~F}, C=10 \times 10^{-20} \mathrm{~F}$, $R_{11}=3 \times 10^{9} \Omega, R_{12}=2 \times 10^{9} \Omega, R_{21}=1 \times 10^{9} \Omega, R_{22}=2 \times 10^{9} \Omega, R=20 \times 10^{9} \Omega$. 


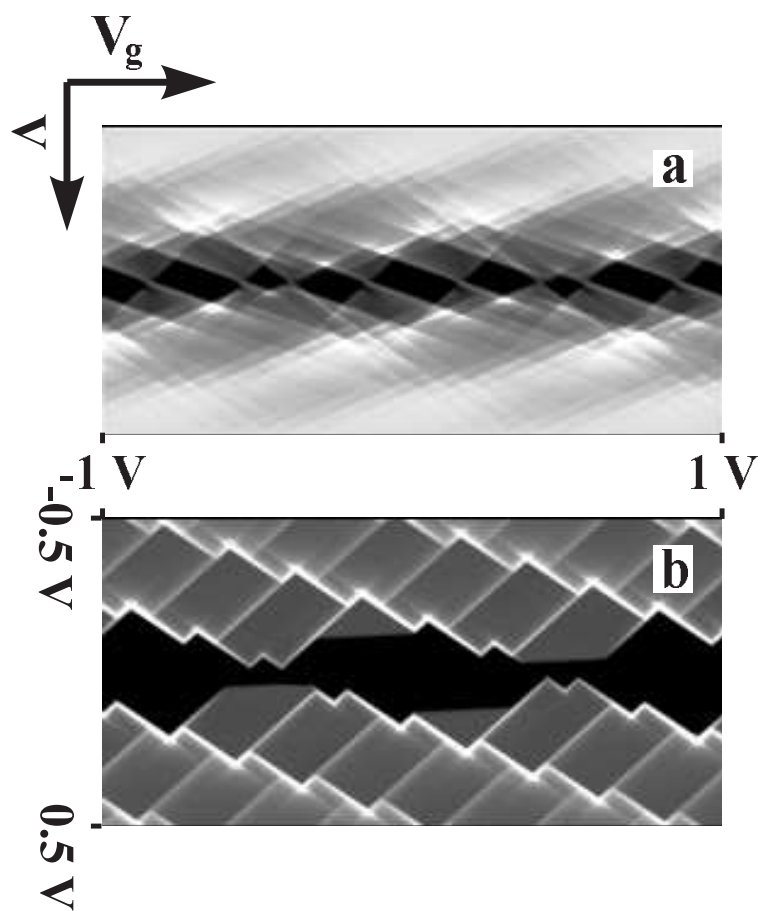

FIG. 3. a.- Double dot system with identical tunneling conductances: $G_{11}=G_{12}=G_{21}=G_{22}=G$. There are five distinct staircase slopes. b.- Every grain has one well-conducting tunneling gap which governs the grain charge: $G_{11}=G_{22}=30 G_{\text {; }}$ $G_{12}=G_{21}=0$. All but two staircase families are suppressed. Same capacitors for symmetric and asymmetric systems: $C_{11}=45 \times 10^{-20} \mathrm{~F}, C_{12}=6 \times 10^{-20} \mathrm{~F}, C_{21}=18 \times 10^{-20} \mathrm{~F}, C_{22}=126 \times 10^{-20} \mathrm{~F}, C_{g 1}=19 \times 10^{-20} \mathrm{~F}, C_{g 2}=47 \times 10^{-20} \mathrm{~F}$, $C=37 \times 10^{-20} \mathrm{~F}$.
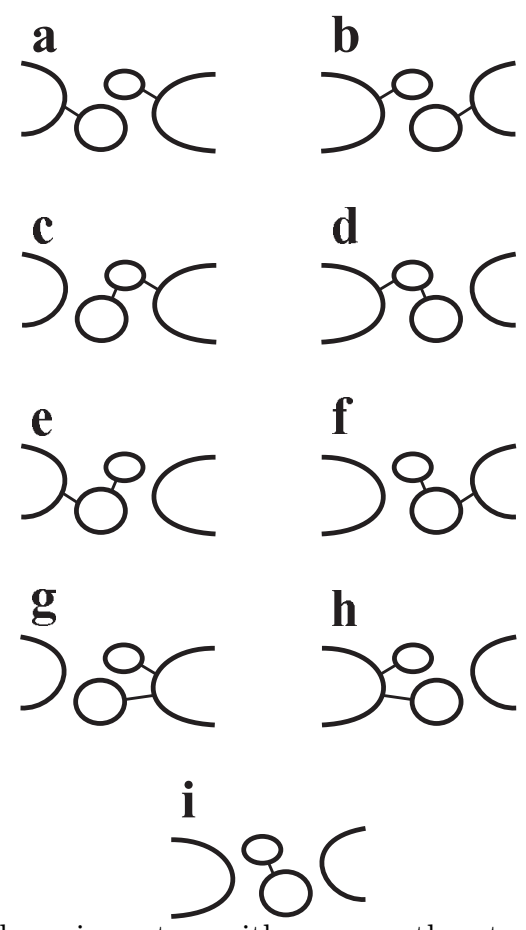

FIG. 4. All possible topologies for a double grain system with no more than two staircase families. The case a produces two families, associated with tunneling gaps $G_{21}$ and $G_{12}$, the case $\mathbf{b}$ - families associated with $G_{11}$ and $G_{22}$, and so on. 

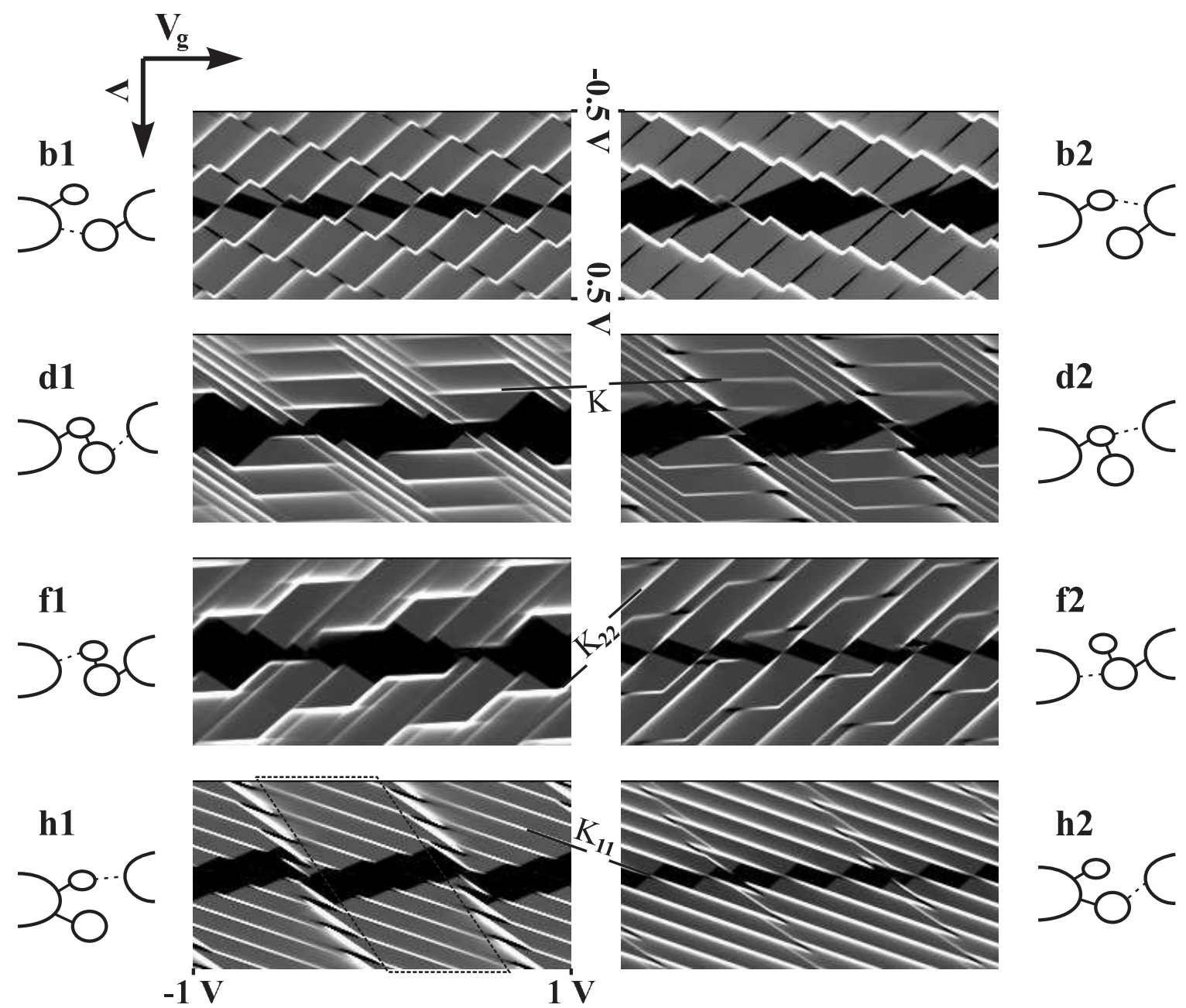

FIG. 5. $\mathrm{dI} / \mathrm{dV}\left(\mathrm{V}, \mathrm{V}_{\mathrm{g}}\right)$ patterns for a double-dot SET: all possible cases (together with Fig. Bb) when just two staircase families are present. For any topology sketch one solid line indicates the most conducting link for the bigger grain and another solid line - the most conducting link for the smaller grain. The dotted line shows the main current channel. Note that in cases b1 and $\mathbf{b 2}$ the second grain gives the staircases with a negative differential conductance. There are also small areas of negative $\mathrm{d} I / \mathrm{d} V$ in $\mathbf{d} \mathbf{2}, \mathbf{f} \mathbf{2}, \mathbf{h} \mathbf{1}$, and $\mathbf{h} \mathbf{2}$.

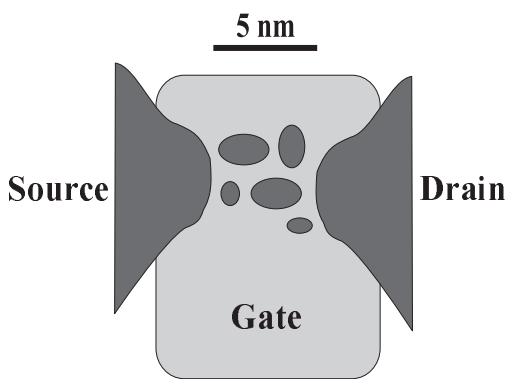

FIG. 6. Sample geometry: Thick low resistive electrodes are bridged by $5 \times 5 \mathrm{~nm}^{2}$ network of metallic clusters. Beneath is an electrostatic gate electrode made of the oxidized Aluminium. 


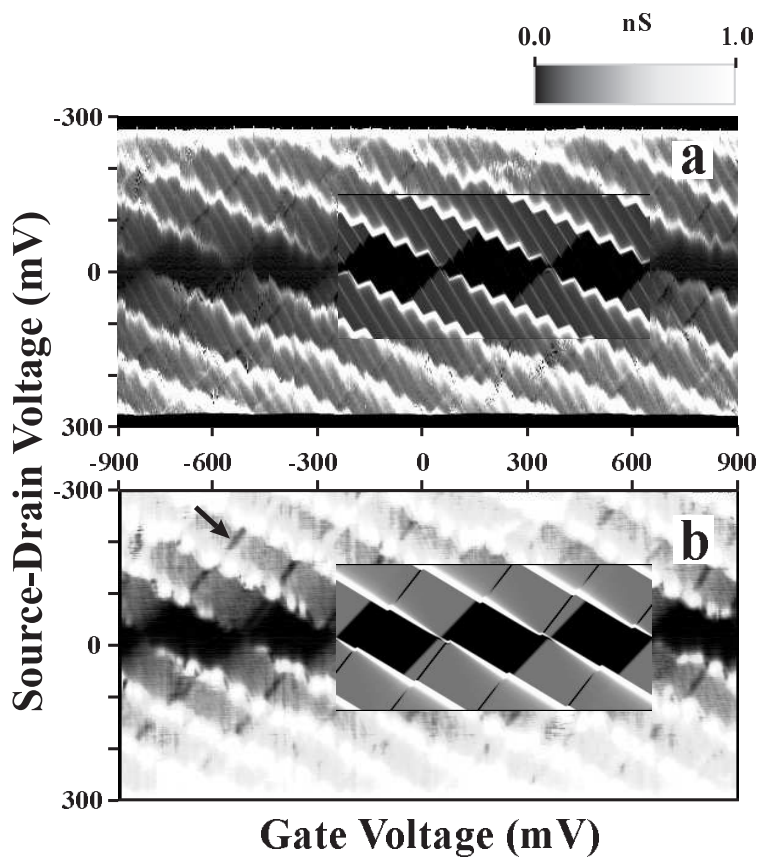

FIG. 7. A typical example of experimental data with the corresponding numeric fit. a.- Two grains connected in series. The widest tunneling gap is between the small grain and the electrode - the same topology as in Fig. 5f 1 . Inset: a numeric fit with parameters $G_{21}=G=30 G_{12} ; G_{11}=G_{22}=0 ; C_{11}=8 \times 10^{-20} \mathrm{~F}, C_{12}=134 \times 10^{-20} \mathrm{~F}, C_{21}=380 \times 10^{-20} \mathrm{~F}$, $C_{22}=114 \times 10^{-20} \mathrm{~F}, C_{g 1}=53 \times 10^{-20} \mathrm{~F}, C_{g 2}=352 \times 10^{-20} \mathrm{~F}, C=41 \times 10^{-20} \mathrm{~F}$. b.- The same sample as in a. Current gradient across the direction indicated by an arrow was computed to highlight the third staircase family almost unresolvable in a. The third grain is coupled the same way as in Fig. 5 b2. Inset: a numeric fit with parameters $G_{11}=G_{22}=30 G_{12}$; $G_{21}=G=0 ; C_{11}=45 \times 10^{-20} \mathrm{~F}, C_{12}=120 \times 10^{-20} \mathrm{~F}, C_{21}=37 \times 10^{-20} \mathrm{~F}, C_{22}=44 \times 10^{-20} \mathrm{~F}, C_{g 1}=53 \times 10^{-20} \mathrm{~F}$, $C_{g 2}=65 \times 10^{-20} \mathrm{~F}, C=7 \times 10^{-20} \mathrm{~F}$.

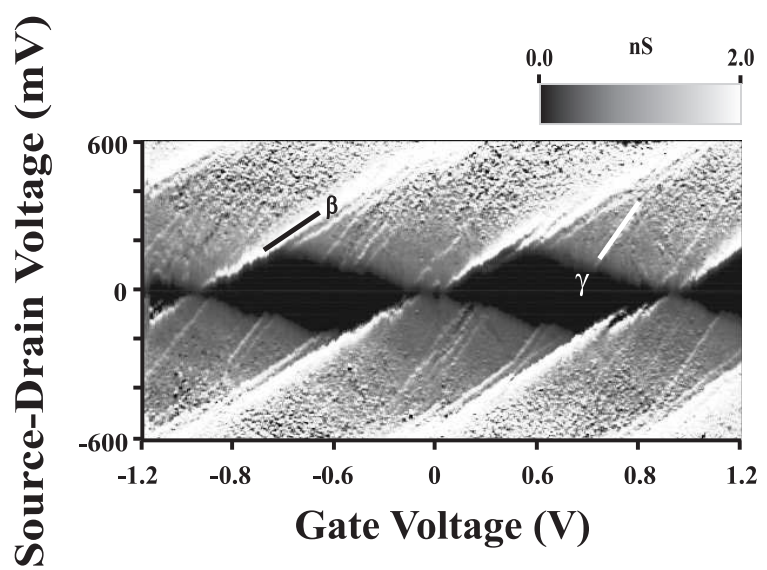

FIG. 8. SET with a charging energy $\sim 90 \mathrm{meV}$. Non-orthodox lines $\gamma$ cannot be attributed to the recharging of some other grains. 